\title{
Free Vibration of Composite Thin-Walled Beams with Chordwise Asymmetric Closed Cross-Sections
}

\author{
Keun-Taek Kim \\ PhD, Principal Researcher, Korea Aerospace Research Institute, Republic of Korea \\ Correspondence should be addressed to Keun-Taek Kim; ktkim@kari.re.kr
}

Received 8 July 2018; Accepted 18 December 2018; Published 25 February 2019

Academic Editor: Maj D. Mirmirani

Copyright (c) 2019 Keun-Taek Kim. This is an open access article distributed under the Creative Commons Attribution License, which permits unrestricted use, distribution, and reproduction in any medium, provided the original work is properly cited.

\begin{abstract}
In this paper, some analytical results via extended Galerkin method on free vibration characteristics of an anisotropic composite beam, which is modeled as a nonuniform thin-walled structure with a chordwise asymmetric closed cross-section and corrected the warping functions, are newly presented. For this study, nonclassical parameters such as warping restraint, transverse shear flexibility, and structural couplings induced by two special configurations, such as circumferentially uniform stiffness (CUS) and circumferentially asymmetric stiffness (CAS), are incorporated. And also, design parameters of the beam associated with preset angles, pretwist angles, taper ratios, and section ratios are additionally investigated. The results of this study could play an important role in more efficient designs of composite thin-walled beams.
\end{abstract}

\section{Introduction}

In the theoretical explicit analyses of thin-walled beams, the closed cross-sections of most aircraft wings and rotor blades have been using almost symmetrical contours in the related literatures based on the author's knowledge [1-9]. A thin-walled beam theory originally developed by Song in his doctoral dissertation [2] has been extensively used in the literature for the study of dynamic response $[3,5,6]$, structural control $[4,7]$, and static aeroelasticity $[8,9]$.

However, in general, aircraft wings have an asymmetrical cross-section in the chordwise direction. Therefore, in this study, we investigated cross-sectional characteristics through the mathematical modeling of asymmetric cross-section and the correction of the warping function. In order to compensate the warping function of the asymmetric cross-section in the chordwise direction, the shape function of the crosssection contour is derived on the basis of an arbitrary pole of the cross-section and the shape function based on the pole is determined so as to satisfy the equilibrium conditions of the warping constraint. And then, the relation of the geometric cross-section is modified based on the pole of the new position and the warping function is corrected and applied to the governing equation.

In this paper, the beam modeled as a chordwise asymmetric cross-section is analyzed for free vibration characteristics numerically, and in order to investigate the effect of the asymmetry of the cross-section, a comparative study was conducted on a symmetrical cross-section model with the same chordwise length and cross-section thickness as a special case of the considered model. In the mathematical modeling, warping restraint, transverse shear flexibility, and structural couplings induced by circumferentially uniform stiffness (CUS) and circumferentially asymmetric stiffness (CAS) configurations $[1,4]$ are considered.

In addition, the present paper examined the effects of the following parameters, such as preset angle, pretwist angle, taper ratio, and section ratio on the eigenfrequencies of the beam. Also, we compared the case where the warping function was corrected and the case where the warping function was not corrected. As a result, in this study, numerical examples were carefully analyzed and compared to investigate the effect of various design parameters on the natural frequencies that provide the basis for dynamic characteristics of beams. 


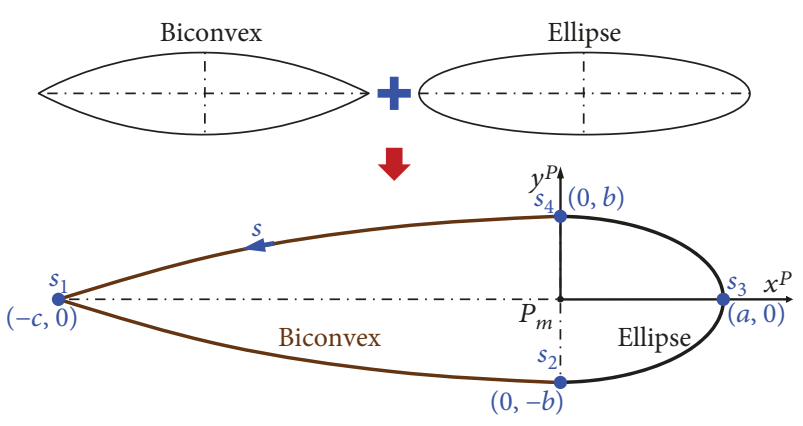

Figure 1: A chordwise asymmetric closed cross-section.

The results presented as families of curves or tables, which may be used for the direct estimation of the eigenfrequencies for any given beam.

\section{Mathematical Modeling}

2.1. Basic Assumptions. The following basic assumptions have been used in this study $[1,2,4,9]$ :

(i) The cross-sections do not deform within their own planes, i.e., no in-plane deformations are allowed

(ii) Transverse shear effects are incorporated. Moreover, the transverse shear strains $\gamma_{x y}$ and $\gamma_{y z}$ are uniform over the entire beam cross-section

(iii) Although the rate of twist is assumed as constant in Saint-Venant torsion, it cannot be assumed to be constant along the beam axis while a warping restraint exists but it has been assumed to be a function of the axis coordinates in this paper

(iv) Primary and secondary warping effects are sufficiently important to be included. The first one is related to the warping displacement of points on the midline cross-section and the second one is related to the points off the midline contour

(v) In the absence of an internal pressure field, the resultant hoop stress is negligibly small as compared to the remaining stresses

(vi) The beam has a nonuniform and asymmetric laminar flow airfoil profile, and in this sense, the beam is considered to be linearly tapered in both the horizontal and vertical planes

(vii) Deformations are small and linear elasticity theory has been applied

2.2. Geometric Configuration of the Model. In this study, Figure 1 shows a chordwise asymmetric closed cross-section of a composite thin-walled cantilevered beam composed of 2 contours, that is, biconvex and ellipse. The pole $\left(P_{m}\right)$ of the section is located at connection points of the 2 different contours on the chord line.

And also, the considered beam in Figure 2 is modeled as an aircraft wing with preset angle $\left(\alpha_{0}\right.$, so-called initial angle

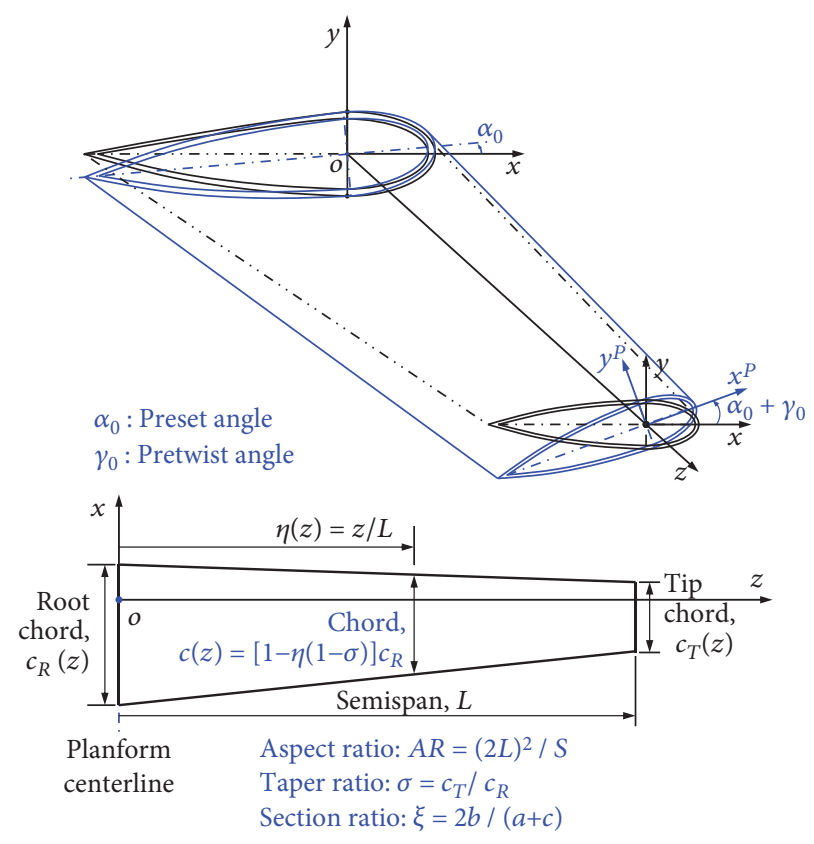

FIgURE 2: The mathematical model.

of attack), pretwist angle $\left(\gamma_{0}\right)$, taper ratio $(\sigma)$, and section ratio $(\xi)$ for the analysis.

2.3. Governing Equations. In principle of the theory for beams, shells and plates, and massive bodies by using the equations of three-dimensional continuum theory should be able to derive with taking advantage of the factors which serve to distinguish each type of structure. In this sense, the theory of plates and shells constitutes a two-dimensional approximation of the three-dimensional elasticity theory, while solid cross-section and thin/thick-walled beams are both one-dimensional approximations of the three-dimensional continuum theory $[1,2]$.

Therefore, using the equations of the 3-D continuum theory, the considered thin-walled beam model was induced to $1-\mathrm{D}$ equations as follows but omitted the process of the transformation in this paper; see reference [1].

$$
\begin{aligned}
u(x, y, z, t)= & u_{0}(z, t)-\left[y(s, z)-n \frac{d x}{d s}\right] \phi(z, t), \\
v(x, y, z, t)= & v_{0}(z, t)-\left[x(s, z)-n \frac{d y}{d s}\right] \phi(z, t), \\
w(x, y, z, t)= & w_{0}(z, t)+x(s, z) \theta_{y}(z, t) \\
& +y(s, z) \theta_{x}(z, t)-F_{w}(s, z) \phi^{\prime}(z, t) \\
& +n\left[\frac{d y}{d s} \theta_{y}(z, t)-\frac{d x}{d s} \theta_{x}(z, t)-a(s, z) \phi^{\prime}(z, t)\right],
\end{aligned}
$$

where $s$ denotes the arc length measured along the circumferential coordinate (whose origin is arbitrarily but conveniently 


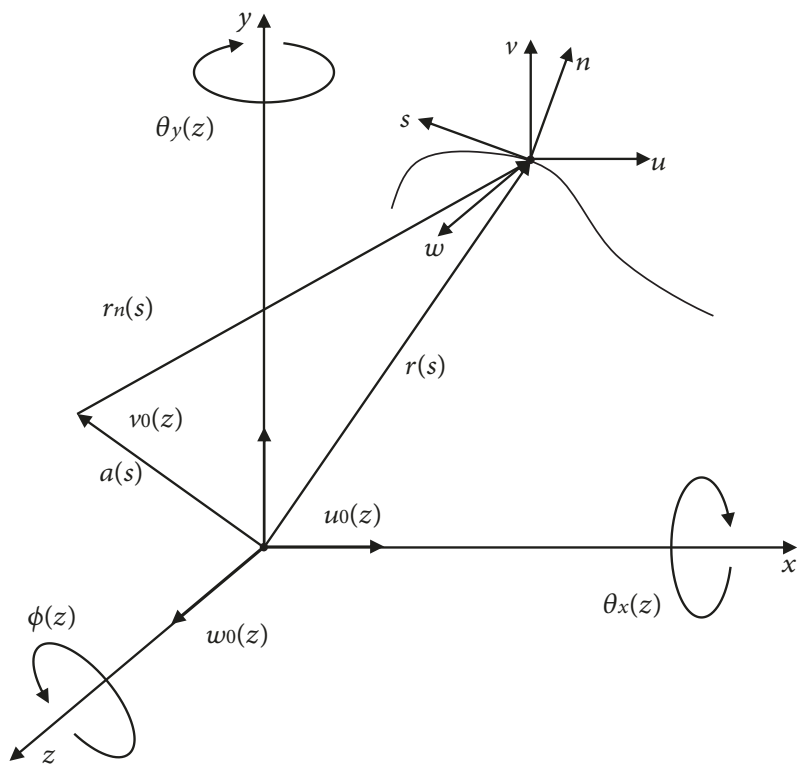

FIgURe 3: The coordinate system.

chosen) and $n$ denotes the coordinate in the thickness direction. In addition,

$$
\begin{aligned}
\theta_{x}(z, t) & =\gamma_{y z}(z, t)-v_{0}^{\prime}(z, t), \\
\theta_{y}(z, t) & =\gamma_{x z}(z, t)-u_{0}^{\prime}(z, t), \\
F_{w}(s, z) & =\int_{0}^{s}\left[r_{n}(s, z)-\psi(s, z)\right] d s=F_{1}(s), \\
a(s, z) & =-x(s, z) \frac{d x}{d s}-y(s, z) \frac{d y}{d s}=F_{2}(n, s), \\
r_{n}(s, z) & =x(s, z) \frac{d y}{d s}-y(s, z) \frac{d x}{d s}, \\
\psi(s, z) & =\frac{\oint\left[r_{n}(s, z)\right] d s}{\oint d s: \text { torsional function }},
\end{aligned}
$$

where $\oint(\cdot) d s$ is the integral along the closed midline contour and $r_{n}(s)=x(s) l+y(s) m$ is a geometric quantity (see Figure 3), where $l=\cos (n, x), m=\sin (n, x)$ denote direction cosines. Also, $F_{1}(s)$ is the primary warping function and $F_{2}$ $(n, s)$ is the secondary warping function.

The boundary value problem, which consists of differential equations and boundary conditions, can be conveniently derived using the extended Hamilton's principle, which is as follows:

$$
\begin{aligned}
& \int_{t_{1}}^{t_{2}}[\delta T-\delta V+\delta W] d t=0, \\
& \delta u_{0}=\delta v_{0}=\delta w_{0}=\delta \theta_{y}=\delta \theta_{x}=\delta \phi=0 \text { at } t=t_{1}, t_{2},
\end{aligned}
$$

where $\delta T$ and $\delta V$ denote the kinetic energy and strain energy, respectively, and $\delta W$ is the virtual work due to nonconservative forces.
TABLE 1: Material properties and geometrical dimensions.

$E_{1}=206.75 \mathrm{GPa}, E_{2}=E_{3}=5.17 \mathrm{GPa}, G_{1}=3.10 \mathrm{GPa}, G_{2}=G_{3}=$ $2.55 \mathrm{GPa}$

$v_{21}=v_{31}=0.00625, v_{32}=0.25, \rho=1528.15 \mathrm{~kg} / \mathrm{m}^{3}$

Chord length: $c+a=0.2 \mathrm{~m}$, section thickness: $b=\xi * c$

Semispan: $L=2.0 \mathrm{~m}$, section contour thickness: $h=0.004 \mathrm{~m}$

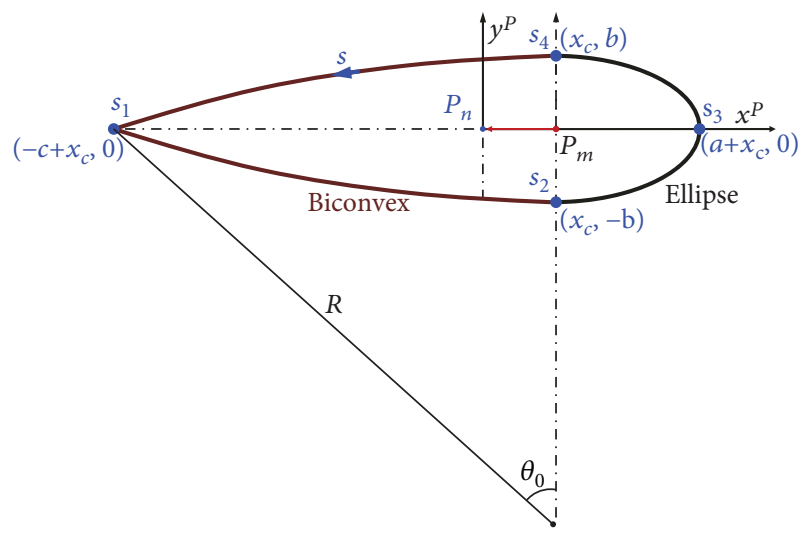

FIgURE 4: The translated geometrical parameters of the section (Model A).

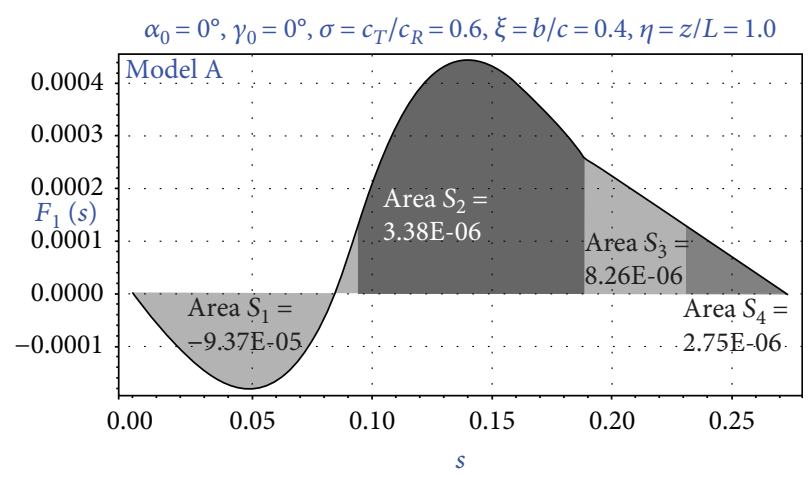

Figure 5: Noncorrected primary warping functions of Model A.

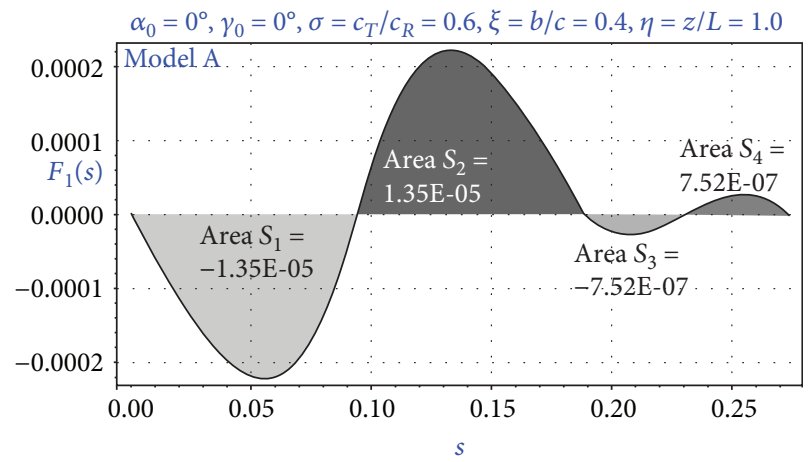

Figure 6: Corrected primary warping functions of Model A.

As a result, the governing equations and boundary conditions are as follows but also omitted the process of the transformation in this paper; see reference [1]. 


\subsubsection{Governing Equations.}

$$
\begin{aligned}
& \delta u_{0}:\left[a_{41} w_{0}^{\prime}+a_{42} \theta_{y}^{\prime}+a_{43} \theta_{x}^{\prime}+a_{44}\left(\theta_{y}+u_{0}^{\prime}\right)\right. \\
& \left.+a_{45}\left(\theta_{x}+v_{0}^{\prime}\right)-a_{46} \phi^{\prime \prime}+a_{47} \phi^{\prime}\right]^{\prime}-\left(b_{1} \ddot{u}_{0}-b_{2} \ddot{\phi}\right)=0, \\
& \delta v_{0}:\left[a_{51} w_{0}^{\prime}+a_{52} \theta_{y}^{\prime}+a_{53} \theta_{x}^{\prime}+a_{54}\left(\theta_{y}+u_{0}^{\prime}\right)\right. \\
& \left.+a_{55}\left(\theta_{x}+v_{0}^{\prime}\right)-a_{56} \phi^{\prime \prime}+a_{57} \phi^{\prime}\right]^{\prime}-\left(b_{1} \ddot{v}_{0}+b_{3} \ddot{\phi}\right)=0, \\
& \delta w_{0}:\left[a_{11} w_{0}^{\prime}+a_{12} \theta_{y}^{\prime}+a_{13} \theta_{x}^{\prime}+a_{14}\left(\theta_{y}+u_{0}^{\prime}\right)+a_{15}\left(\theta_{x}+v_{0}^{\prime}\right)\right. \\
& \left.-a_{16} \phi^{\prime \prime}+a_{17} \phi^{\prime}\right]^{\prime}-\left(b_{1} \ddot{w}_{0}+b_{3} \ddot{\theta}_{y}+b_{2} \ddot{\theta}_{x}-b_{7} \ddot{\phi}\right)=0 \text {, } \\
& \delta \theta_{y}:\left[a_{21} w_{0}^{\prime}+a_{22} \theta_{y}^{\prime}+a_{23} \theta_{x}^{\prime}+a_{24}\left(\theta_{y}+u_{0}^{\prime}\right)\right. \\
& \left.+a_{25}\left(\theta_{x}+v_{0}^{\prime}\right)-a_{26} \phi^{\prime \prime}+a_{27} \phi^{\prime}\right]^{\prime}-\left[a_{41} w_{0}^{\prime}\right. \\
& +a_{42} \theta_{y}^{\prime}+a_{43} \theta_{x}^{\prime}+a_{44}\left(\theta_{y}+u_{0}^{\prime}\right)+a_{45}\left(\theta_{x}+v_{0}^{\prime}\right) \\
& \left.-a_{46} \phi^{\prime \prime}+a_{47} \phi^{\prime}\right]-\left(b_{3} \ddot{w}_{0}+b_{5} \ddot{\theta}_{y}+b_{6} \ddot{\theta}_{x}-b_{9} \ddot{\phi}^{\prime}\right. \\
& \left.+b_{15} \ddot{\theta}_{y}-b_{13} \ddot{\theta}_{x}-b_{17} \ddot{\phi}^{\prime}\right)=0 \text {, } \\
& \delta \theta_{x}:\left[a_{31} w_{0}^{\prime}+a_{32} \theta_{y}^{\prime}+a_{33} \theta_{x}^{\prime}+a_{34}\left(\theta_{y}+u_{0}^{\prime}\right)\right. \\
& \left.+a_{35}\left(\theta_{x}+v_{0}^{\prime}\right)-a_{36} \phi^{\prime \prime}+a_{37} \phi^{\prime}\right]^{\prime}-\left[a_{51} w_{0}^{\prime}\right. \\
& +a_{52} \theta_{y}^{\prime}+a_{53} \theta_{x}^{\prime}+a_{54}\left(\theta_{y}+u_{0}^{\prime}\right)+a_{55}\left(\theta_{x}+v_{0}^{\prime}\right) \\
& \left.-a_{56} \phi^{\prime \prime}+a_{57} \phi^{\prime}\right]-\left(b_{2} \ddot{w}_{0}+b_{6} \ddot{\theta}_{y}+b_{4} \ddot{\theta}_{x}-b_{8} \ddot{\phi}^{\prime}\right. \\
& \left.-b_{13} \ddot{\theta}_{y}+b_{14} \ddot{\theta}_{x}+b_{16} \ddot{\phi}^{\prime}\right)=0 \text {, } \\
& \delta \phi:\left[a_{71} w_{0}^{\prime}+a_{72} \theta_{y}^{\prime}+a_{73} \theta_{x}^{\prime}+a_{74}\left(\theta_{y}+u_{0}^{\prime}\right)\right. \\
& \left.+a_{75}\left(\theta_{x}+v_{0}^{\prime}\right)-a_{76} \phi^{\prime \prime}+a_{77} \phi^{\prime}\right]^{\prime}-\left[a_{61} w_{0}^{\prime}\right. \\
& +a_{62} \theta_{y}^{\prime}+a_{63} \theta_{x}^{\prime}+a_{64}\left(\theta_{y}+u_{0}^{\prime}\right)+a_{65}\left(\theta_{x}+v_{0}^{\prime}\right) \\
& \left.-a_{66} \phi^{\prime \prime}+a_{67} \phi^{\prime}\right]-\left(b_{4}+b_{5}+b_{14}+b_{15}\right) \ddot{\phi} \\
& -b_{3} \ddot{v}_{0}+b_{2} \ddot{u}_{0}-\left(-b_{7} \ddot{w}_{0}-b_{9} \ddot{\theta}_{y}-b_{8} \ddot{\theta}_{x}\right. \\
& \left.+b_{10} \ddot{\phi}^{\prime}-b_{17} \ddot{\theta}_{y}+b_{16} \ddot{\theta}_{x}+b_{18} \ddot{\phi}^{\prime}\right)=0 \text {. }
\end{aligned}
$$

\subsubsection{Boundary Conditions.}

$$
\begin{gathered}
\delta u_{0}: a_{41} w_{0}^{\prime}+a_{42} \theta_{y}^{\prime}+a_{43} \theta_{x}^{\prime}+a_{44}\left(\theta_{y}+u_{0}^{\prime}\right) \\
+a_{45}\left(\theta_{x}+v_{0}^{\prime}\right)-a_{46} \phi^{\prime \prime}+a_{47} \phi^{\prime}=0 \\
\delta v_{0}: a_{51} w_{0}^{\prime}+a_{52} \theta_{y}^{\prime}+a_{53} \theta_{x}^{\prime}+a_{54}\left(\theta_{y}+u_{0}^{\prime}\right) \\
+a_{55}\left(\theta_{x}+v_{0}^{\prime}\right)-a_{56} \phi^{\prime \prime}+a_{57} \phi^{\prime}=0,
\end{gathered}
$$

$$
\begin{aligned}
\delta w_{0} & : a_{11} w_{0}^{\prime}+a_{12} \theta_{y}^{\prime}+a_{13} \theta_{x}^{\prime}+a_{14}\left(\theta_{y}+u_{0}^{\prime}\right) \\
+ & a_{15}\left(\theta_{x}+v_{0}^{\prime}\right)-a_{16} \phi^{\prime \prime}+a_{17} \phi^{\prime}=0 \\
\delta \theta_{y} & : a_{21} w_{0}^{\prime}+a_{22} \theta_{y}^{\prime}+a_{23} \theta_{x}^{\prime}+a_{24}\left(\theta_{y}+u_{0}^{\prime}\right) \\
& +a_{25}\left(\theta_{x}+v_{0}^{\prime}\right)-a_{26} \phi^{\prime \prime}+a_{27} \phi^{\prime}=0, \\
\delta \theta_{x} & : a_{31} w_{0}^{\prime}+a_{32} \theta_{y}^{\prime}+a_{33} \theta_{x}^{\prime}+a_{34}\left(\theta_{y}+u_{0}^{\prime}\right) \\
& +a_{35}\left(\theta_{x}+v_{0}^{\prime}\right)-a_{36} \phi^{\prime \prime}+a_{37} \phi^{\prime}=0, \\
\delta \phi & : a_{71} w_{0}^{\prime}+a_{72} \theta_{y}^{\prime}+a_{73} \theta_{x}^{\prime}+a_{74}\left(\theta_{y}+u_{0}^{\prime}\right) \\
& +a_{75}\left(\theta_{x}+v_{0}^{\prime}\right)-a_{76} \phi^{\prime \prime}+a_{77} \phi^{\prime}-\left[a_{61} w_{0}^{\prime}+a_{62} \theta_{y}^{\prime}\right. \\
& +a_{63} \theta_{x}^{\prime}+a_{64}\left(\theta_{y}+u_{0}^{\prime}\right)+a_{65}\left(\theta_{x}+v_{0}^{\prime}\right)-a_{66} \phi^{\prime \prime} \\
& \left.+a_{67} \phi^{\prime}\right]^{\prime}-\left(-b_{7} \ddot{w}_{0}-b_{9} \ddot{\theta}_{y}-b_{8} \ddot{\theta}_{x}+b_{10} \ddot{\phi}^{\prime}\right. \\
& \left.+b_{17} \ddot{\theta}_{y}+b_{16} \ddot{\theta}_{x}+b_{18} \ddot{\phi}^{\prime}\right)=0 \\
\delta \phi^{\prime} & : a_{61} w_{0}^{\prime}+a_{62} \theta_{y}^{\prime}+a_{63} \theta_{x}^{\prime}+a_{64}\left(v_{y}^{\prime}+u_{0}^{\prime}\right)-a_{66} \phi^{\prime \prime}+a_{67} \phi^{\prime}=0, \\
&
\end{aligned}
$$

where $a_{i j}(i, j=1,2, \ldots, 6)$ are stiffness coefficients and $b_{j}(j=1,2, \ldots, 18)$ are mass coefficients.

\section{Numerical Analysis and Discussion}

3.1. Material Properties and Geometrical Dimensions. Wing structures modeled as a cantilevered thin-walled beam of a chordwise asymmetric closed cross-section made of T300/5208 carbon epoxy material are considered for numerical simulations. Their geometrical dimensions and material properties are shown in Table 1.

3.2. Correction of the Primary Warping Functions. The warping displacement in pure twist can be obtained as the product of the warping function and the rate of twist $[1,2]$.

$$
w(s, z, t)=F_{1}(s) \phi^{\prime}(z, t)
$$

where $F_{1}(s)=\int_{0}^{s}\left(\lambda-r_{n}\right) d s+C$, the constant $C$ being determined by satisfying the following warping equilibrium condition:

$$
\oint F_{1}(s) d s=0,
$$

thus yielding

$$
C=\frac{\oint F_{1} d s}{\oint d s} .
$$


TABle 2: Mass coefficients of Model A and Model C for CUS and CAS.

\begin{tabular}{lcccccccccccccccccc}
\hline & $b_{1}$ & $b_{2}$ & $b_{3}$ & $b_{4}$ & $b_{5}$ & $b_{6}$ & $b_{7}$ & $b_{8}$ & $b_{9}$ & $b_{10}$ & $b_{11}$ & $b_{12}$ & $b_{13}$ & $b_{14}$ & $b_{15}$ & $b_{16}$ & $b_{17}$ & $b_{18}$ \\
\hline$\alpha_{0}=0$ and $\gamma_{0}=0$ & $S$ & & $A$ & $S$ & $S$ & & & $A$ & & $S$ & & & $S$ & $S$ & $S$ & $A$ & & $S$ \\
$\alpha_{0} \neq 0$ or $\gamma_{0} \neq 0$ & $S$ & $A$ & $A$ & $S$ & $S$ & $S$ & & $A$ & $A$ & $S$ & & & $S$ & $S$ & $S$ & $A$ & $A$ & $S$ \\
\hline
\end{tabular}

In equation $(7), \oint(\cdot) d s$ denotes the integration around the whole midline contour of the cross-section.

Note that fulfillment of $\oint F_{1} d s=0$ implies

$$
\oint_{K} \frac{\partial w}{\partial s} d s=0, \quad(K=1,2, \ldots, N),
$$

and vice-versa [1].

From the above relations, equations (6)-(9), the warping functions could be corrected for the model (see Figure 4) as the following procedures.

Step 1. In advance, the geometrical shape functions of the contour are derived as follows:

For an ellipse contour, from $x^{2} / a^{2}+y^{2} / b^{2}=1$,

$$
\begin{aligned}
& x= \pm a \cos \varphi, \\
& y= \pm b \sin \varphi .
\end{aligned}
$$

For a biconvex contour, from $x^{2}+\left(y+R \cos \theta_{0}\right)^{2}=R^{2}$ at the upper,

$$
\begin{aligned}
& x= \pm R \sin \varphi, \\
& y= \pm R \cos \varphi-R \cos \theta_{0} .
\end{aligned}
$$

And from $x^{2}+\left(y-R \cos \theta_{0}\right)^{2}=R^{2}$ at the lower,

$$
\begin{aligned}
& x= \pm R \sin \varphi, \\
& y= \pm R \cos \varphi+R \cos \theta_{0} .
\end{aligned}
$$

And also, the length and the area of the contours are

$$
\begin{aligned}
\Omega & =\Omega_{e}+\Omega_{b}, \\
\beta & =\beta_{e}+\beta_{b}, \\
\Omega_{b} & =\frac{R^{2}\left(2 \theta_{0}-\sin \left[2 \theta_{0}\right]\right)}{2}, \\
\Omega_{e} & =\frac{\pi a b}{2}, \\
\beta_{e} & =2 c E_{m}, \\
\beta_{b} & =2 R \theta_{0},
\end{aligned}
$$

where $E_{m}$ is the complete elliptical integral $\left(m=1-b^{2} / a^{2}\right.$, where $a \geq b), \Omega$ and $\beta$ are the all area and all length of the contours, and $(\cdot)_{e}$ and $(\cdot)_{b}$ mean the physical quantities of the ellipse and biconvex contours, respectively.
From the above relations, we have the geometrical shape functions of the contour as follows:

$$
\begin{aligned}
& s_{0} \leq s \leq s_{1}: x_{1}^{P}=-R \sin \left[\frac{s}{R}\right], \\
& y_{1}^{P}=b-R+R \cos \left[\frac{s}{R}\right], \\
& s_{1} \leq s \leq s_{2}: x_{2}^{P}=-R \sin \left[\frac{\left(\beta_{b}-s\right)}{R}\right], \\
& y_{2}^{P}=b-R+R \cos \left[\frac{\left(\beta_{b}-s\right)}{R}\right], \\
& s_{2} \leq s \leq s_{3}: x_{3}^{P}=-R \sin \left[\frac{\pi\left(\beta_{b}-s\right)}{\beta_{e}}\right], \\
& y_{3}^{P}=-b \cos \left[\frac{\pi\left(\beta_{b}-s\right)}{\beta_{e}}\right], \\
& s_{3} \leq s \leq s_{4}: x_{4}^{P}=-R \sin \left[\frac{\pi\left(\beta_{b}-s\right)}{\beta_{e}}\right], \\
& y_{4}^{P}=-b \cos \left[\frac{\pi\left(\beta_{b}-s\right)}{\beta_{e}}\right] .
\end{aligned}
$$

Step 2. And then, for correction of the warping functions, so as to satisfy equilibrium condition (7), the pole $\left(P_{m}\right)$ should be translated to the new one $\left(P_{n}\right)$ and also the shape functions (equation (14)) modified as follows:

$$
\begin{aligned}
& s_{0} \leq s \leq s_{1}: x_{1}^{P}=x_{c}-R \sin \left[\frac{s}{R}\right], \\
& y_{1}^{P}=b-R+R \cos \left[\frac{s}{R}\right], \\
& s_{1} \leq s \leq s_{2}: x_{2}^{P}=x_{c}-R \sin \left[\frac{\left(\beta_{b}-s\right)}{R}\right], \\
& y_{2}^{P}=b-R+R \cos \left[\frac{\left(\beta_{b}-s\right)}{R}\right], \\
& s_{2} \leq s \leq s_{3}: x_{3}^{P}=x_{c}-R \sin \left[\frac{\pi\left(\beta_{b}-s\right)}{\beta_{e}}\right], \\
& y_{3}^{P}=-b \cos \left[\frac{\pi\left(\beta_{b}-s\right)}{\beta_{e}}\right], \\
& s_{3} \leq s \leq s_{4}: x_{4}^{P}=x_{c}-R \sin \left[\frac{\pi\left(\beta_{b}-s\right)}{\beta_{e}}\right], \\
& y_{4}^{P}=-b \cos \left[\frac{\pi\left(\beta_{b}-s\right)}{\beta_{e}}\right],
\end{aligned}
$$


Table 3: Stiffness coefficients of Model A and Model C.

(a)

\begin{tabular}{llcccccc}
\hline \multicolumn{7}{c}{ CUS: $\alpha_{0}=0$ and $\gamma_{0}=0$} \\
\hline$a_{i j}$ & 1 & 2 & 3 & 4 & 5 & 6 & 7 \\
1 & $S$ & $A$ & & & & & $S$ \\
2 & & $S$ & & & $S$ & & $A$ \\
3 & & & $S$ & $S$ & & $A$ & \\
4 & & & & $S$ & & $A$ & \\
5 & & & & $S$ & & \\
6 & & & Sym. & & & $S$ & \\
7 & & & & & & $S$ \\
\hline
\end{tabular}

(b)

\begin{tabular}{llcccccc}
\hline \multicolumn{7}{c}{ CAS: $\alpha_{0}=0$ and $\gamma_{0}=0$} \\
\hline$a_{i j}$ & 1 & 2 & 3 & 4 & 5 & 6 & 7 \\
1 & $S$ & $A$ & & $S$ & & & \\
2 & & $S$ & & $A$ & & & \\
3 & & & $S$ & & & $A$ & $S$ \\
4 & & & & $S$ & & & \\
5 & & & & & $S$ & $S$ & \\
6 & & & Sym. & & & $S$ & $A$ \\
7 & & & & & & $S$ \\
\hline
\end{tabular}

(c)

\begin{tabular}{lllccccc}
\hline \multicolumn{7}{c}{ CUS: $\alpha_{0} \neq 0$ or $\gamma_{0} \neq 0$} \\
\hline$a_{i j}$ & 1 & 2 & 3 & 4 & 5 & 6 & 7 \\
1 & $S$ & $S$ & $A$ & & & & $S$ \\
2 & & $S$ & $A$ & & $S$ & $A$ & $S$ \\
3 & & & $S$ & $S$ & & $S$ & $A$ \\
4 & & & & $S$ & $A$ & $S$ & \\
5 & & & & & $S$ & $A$ & \\
6 & & & Sym. & & & $S$ & \\
7 & & & & & & $S$ \\
\hline
\end{tabular}

(d)

\begin{tabular}{llllllll}
\hline \multicolumn{7}{c}{ CAS: $\alpha_{0} \neq 0$ or $\gamma_{0} \neq 0$} \\
\hline$a_{i j}$ & 1 & 2 & 3 & 4 & 5 & 6 & 7 \\
1 & $S$ & $S$ & $A$ & $S$ & $A$ & & \\
2 & & $S$ & $A$ & $S$ & $A$ & $A$ & $A$ \\
3 & & & $S$ & $A$ & $A$ & $S$ & $S$ \\
4 & & & & $S$ & $A$ & $A$ & \\
5 & & & & & $S$ & $S$ & \\
6 & & & Sym. & & & $S$ & $A$ \\
7 & & & & & & $S$ \\
\hline
\end{tabular}
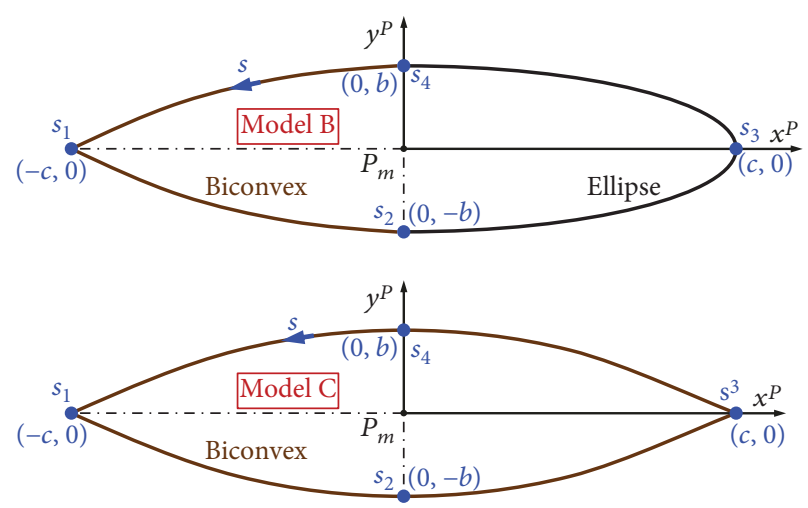

Figure 7: Model B and Model C for comparing.

where $x_{c}$ is a translation quantity along the chordwise (see Figure 4), that is,

$$
x_{c}=\frac{a c \csc \left(\theta_{0} / 2\right)^{2}\left[\begin{array}{c}
\left(-2 E_{m}+\pi\right) \theta_{0}-\pi \theta_{0} \cos \left(\theta_{0}\right) \\
+E_{m} \sin \left(2 \theta_{0}\right)
\end{array}\right]}{\left[4\left(c \theta_{0}\right)+a E_{m} \sin \left(\theta_{0}\right)\right]}
$$

To verify the above results through an example, in case of the noncorrected primary warping functions (equation (14)), the function's graph can be depicted in Figure 5. In Figure 5, the sum of the area based on the $x$-axis is not zero. That means that the warping function should be corrected [1]. Therefore, from the corrected warping functions (equations (15) and (16)), Figure 6 can be drawn and shows that the all area is zero. As a result, the above relations, equations (15) and (16), will be applied to governing equations and boundary conditions of the beam for analysis.

3.3. Characteristics of the Cross-Section. For a survey of the characteristics of the chordwise asymmetric closed crosssection (Model A), using the corrected warping functions, mass coefficients $b_{j}$ and stiffness coefficients $a_{i j}$ of equations (4) and (5) were found. The details of the mass and stiffness coefficients could be found in reference [1], which are equations (4.1-4) for mass coefficients and Tables 4.3-1 for stiffness coefficients of chapter 4 . The results of the nonzero coefficients were provided in Tables 2 and 3 for comparing between Model A and a symmetric one (Model C, see Figure 7$)$ according to a preset angle $\left(\alpha_{0}\right)$ and a pretwist angle $\left(\gamma_{0}\right)$ of CUS and CAS configurations for each. In the tables, " $S$ " entries are valued elements of the symmetric section (Model C) and " $A$ " entries are those of the asymmetric section (Model A) in addition to the results of Model C.

And then, stiffness coefficients $a_{i j}\left(=a_{j i}\right)$ in cases of $\left(\alpha_{0}=0^{\circ}\right.$ and $\left.\gamma_{0}=0^{\circ}\right)$ and $\left(\alpha_{0}=4^{\circ}\right.$ and $\left.\gamma_{0}=10^{\circ}\right)$ were depicted in Figures 8 and 9 for CUS and CAS at $\eta=1.0, \sigma=0.6$, and $\xi=0.4$. These figures show that $a_{14}, a_{15}, a_{24}, a_{25}, a_{27}, a_{34}$, $a_{35}, a_{37}, a_{45}, a_{46}, a_{56}$, and $a_{67}$ have asymmetric values and the others have symmetric values at a ply angle of $90^{\circ}$. 

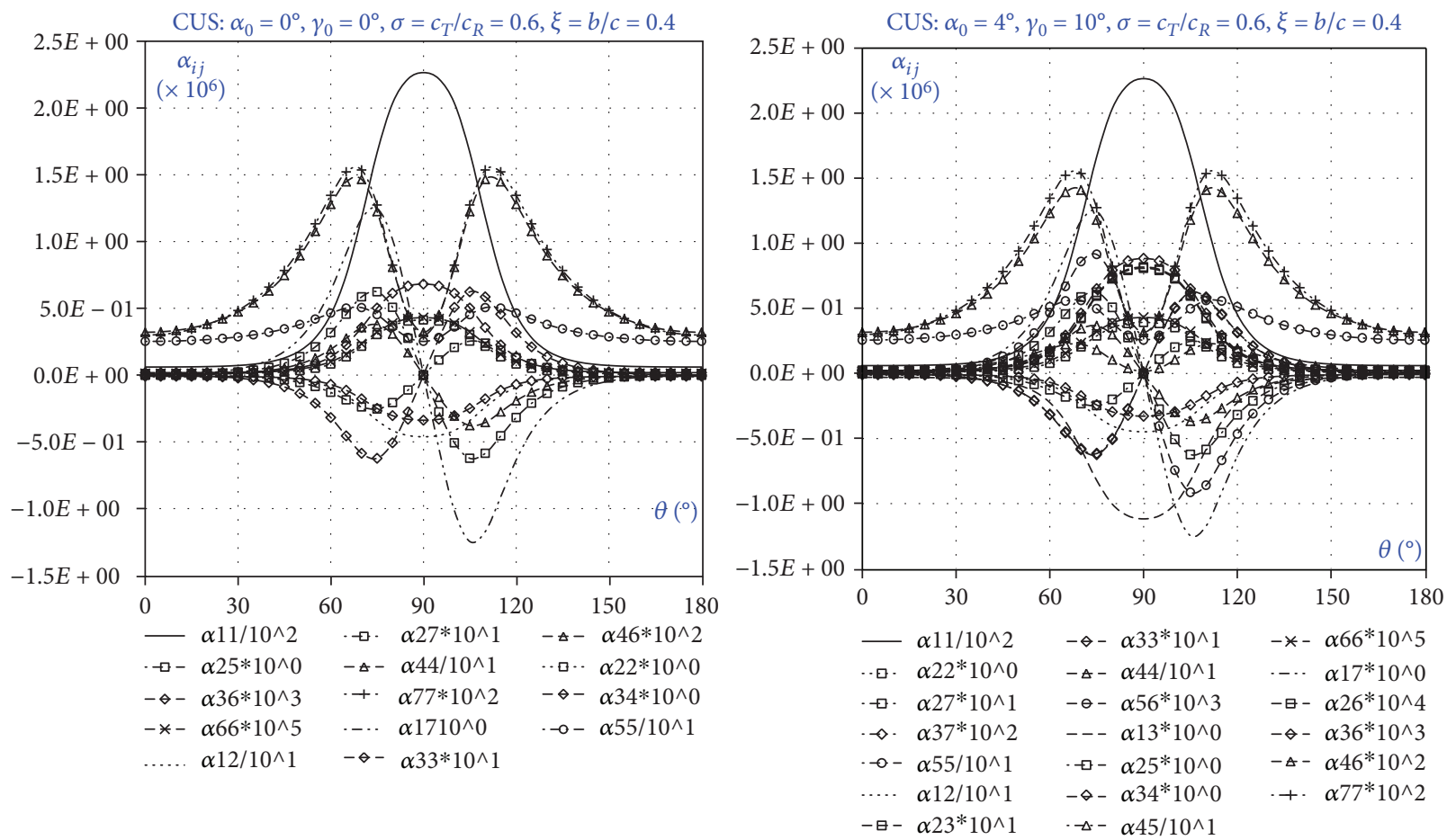

FIgURE 8: Stiffness coefficients $a_{i j}\left(=a_{j i}\right)$ vs. ply angle of Model A for CUS.
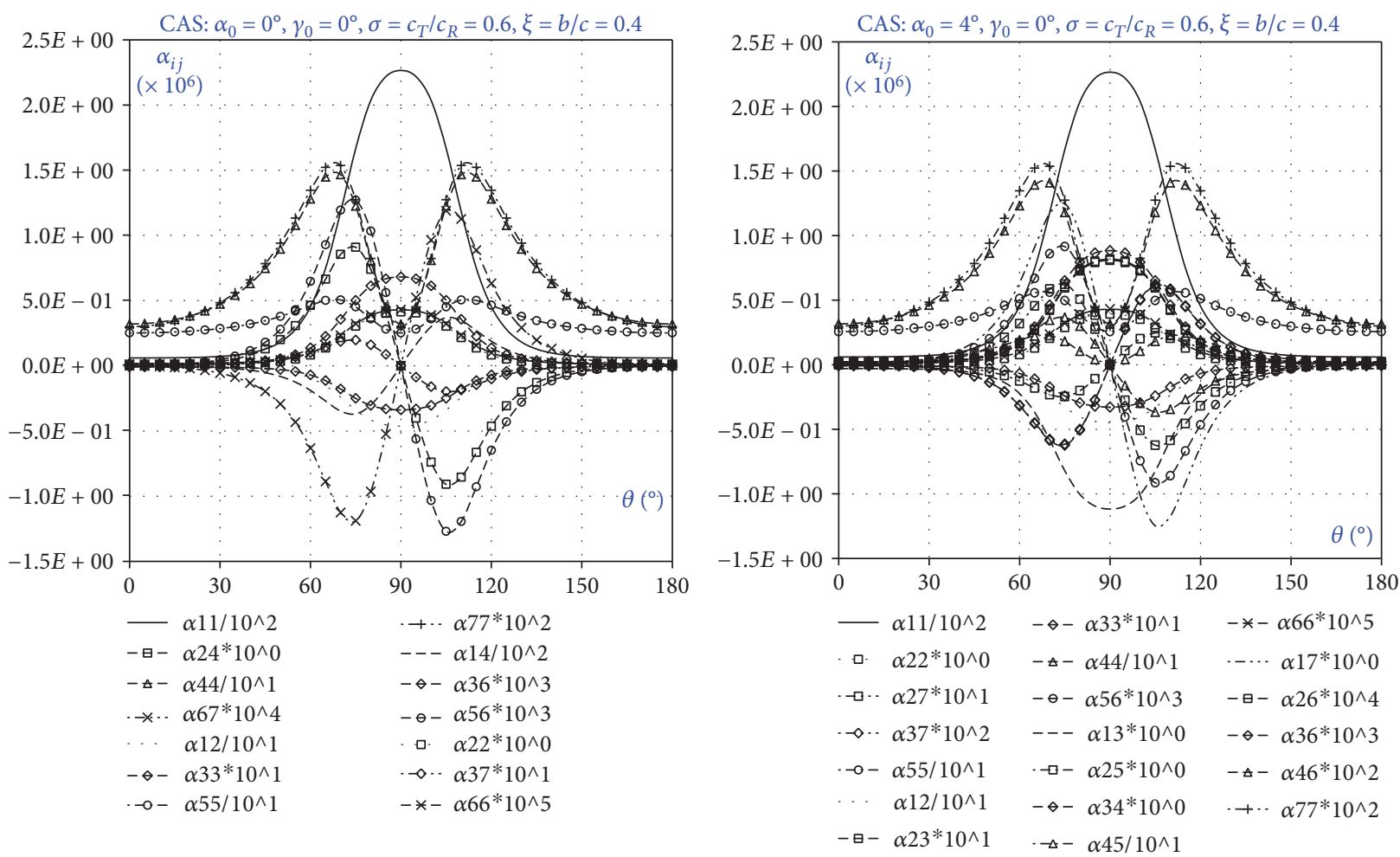

Figure 9: Stiffness coefficients $a_{i j}\left(=a_{j i}\right)$ vs. ply angle of Model A for CAS.

Furthermore, Table 4 shows that some stiffness coefficients of the model have different values between $\alpha_{0}=0^{\circ}$ and $\gamma_{0}=0^{\circ}$ and $\alpha_{0} \neq 0^{\circ}$ or $\gamma_{0} \neq 0^{\circ}$ and some are the same or the others only appeared in case of $\alpha_{0} \neq 0^{\circ}$ or $\gamma_{0} \neq 0^{\circ}$. And especially, when $i=j, a_{i i}$ have all the same values for CUS and CAS configurations. 


\subsection{Eigenfrequency of the Model}

3.4.1. Stiffness and Mass Matrices. The eigenvalue problem is discretized by representing in the form via extended Galerkin method (EGM):

$$
\begin{aligned}
& u_{0}(z, t)=\sum_{j=1}^{N} a_{j} \psi_{j}^{(1)}(z), \\
& v_{0}(z, t)=\sum_{j=1}^{N} b_{j} \psi_{j}^{(2)}(z), \\
& w_{0}(z, t)=\sum_{j=1}^{N} c_{j} \psi_{j}^{(3)}(z), \\
& \theta_{y}(z, t)=\sum_{j=1}^{N} d_{j} \psi_{j}^{(4)}(z), \\
& \theta_{x}(z, t)=\sum_{j=1}^{N} e_{j} \psi_{j}^{(5)}(z), \\
& \phi(z, t)=\sum_{j=1}^{N} f_{j} \psi_{j}^{(6)}(z),
\end{aligned}
$$

where $\psi_{j}^{(r)}$ are trial functions and have to satisfy the geometrical boundary conditions at $z=0$ and also, $a_{j}, b_{j}, c_{j}, d_{j}, e_{j}$, and $f_{j}$ are arbitrary coefficients. Now, the discretization is accomplished directly in the Hamilton's principle functional and then written in a matrix form as follows:

$$
\begin{aligned}
& {\left[\begin{array}{ccc} 
& \cdots & \\
\vdots & K_{p q} & \vdots \\
\cdots &
\end{array}\right]\left\{\begin{array}{c}
a_{j} \\
\vdots \\
f_{j}
\end{array}\right\}} \\
& -\omega^{2}\left[\begin{array}{ccc} 
& \cdots \\
\vdots & M_{p q} & \vdots \\
& \ldots
\end{array}\right]\left\{\begin{array}{c}
a_{j} \\
\vdots \\
f_{j}
\end{array}\right\}=\left\{\begin{array}{c}
Q_{1} \\
\vdots \\
Q_{6}
\end{array}\right\},
\end{aligned}
$$

where $\left[K_{p q}\right]$ and $\left[M_{p q}\right]$ are stiffness and mass matrices, respectively, and $\left\{Q_{r}\right\}(r=1,2, \ldots, 6)$ is a force vector.

Similarly, for the survey of the characteristics of the chordwise asymmetric closed cross-section (Model A), using the corrected warping functions, the mass and stiffness matrices of equation (18) were found. The results of the nonzero coefficients were provided in Tables 5 and 6 for comparing between Model A and a symmetric one (Model C, see Figure 7) according to a preset angle $\left(\alpha_{0}\right)$ and a pretwist angle $\left(\gamma_{0}\right)$ of CUS and CAS configurations for each. In the tables, " $S$ " entries are valued elements of the symmetric section (Model C) and " $A$ " entries are those of the asymmetric section (Model A) in addition to Model C.

At this point, Table 5 shows that the stiffness matrix for CAS configuration is composed of the same elements
Table 4: Nonzero stiffness coefficients of Model A.

\begin{tabular}{lc}
\hline CUS & $a_{i j}\left(=a_{j i}\right)$ \\
Different values & $a_{11}, a_{17}, a_{25}, a_{34}, a_{66}, a_{77}$ \\
Same values & $a_{12}, a_{22}, a_{27}, a_{33}, a_{36}, a_{44}, a_{46}, a_{55}$ \\
Only $\alpha_{0} \neq 0$ or $\gamma_{0} \neq 0$ & $a_{13}, a_{23}, a_{26}, a_{37}, a_{45}, a_{56}$ \\
\hline CAS & $a_{i j}\left(=a_{j i}\right)$ \\
Different values & $a_{11}, a_{66}, a_{67}, a_{77}$ \\
Same values & $a_{12}, a_{14}, a_{22}, a_{24}, a_{33}, a_{36}, a_{37}, a_{44}, a_{55}, a_{56}$ \\
Only $\alpha_{0} \neq 0$ or $\gamma_{0} \neq 0$ & $a_{13}, a_{15}, a_{23}, a_{25}, a_{26}, a_{27}, a_{34}, a_{35}, a_{45}, a_{46}$ \\
\hline
\end{tabular}

between Model A and Model C, in cases of $\alpha_{0}=0$ and $\gamma_{0}=$ 0 and $\alpha_{0} \neq 0$ or $\gamma_{0} \neq 0$. And also, mass matrices have all the same values for CUS and CAS configurations at any case.

3.4.2. Effects of Preset Angle and Pretwist Angle on Eigenfrequency. In order to investigate the effects of preset and pretwist angles on eigenfrequencies calculated for Model A, both CUS and CAS configurations. The results are shown in Figure 10 and Table 7.

In Figure 10 and Table 7, preset and pretwist angles have no near effects on eigenfrequencies; nevertheless, the differences exist but very negligibly small.

3.4.3. Effects of Correction of the Primary Warping Functions. Next, based on ply angles, the effects of corrected (CW) and noncorrected (NCW) warping functions on eigenfrequencies were estimated for Model A, both CUS and CAS configurations. The results are shown in Figure 11 and Table 8.

In Figure 11 and Table 8, the effects of corrected (CW) and noncorrected (NCW) warping functions on eigenfrequencies were observed to have very small differences for ply angles. However, for precise calculation, the primary warping function should be properly corrected according to the warping equilibrium condition from equations (6)-(9).

3.4.4. Effects of Geometrical Parameters. From now on, the effects of geometrical parameters $\left(\alpha_{0}, \gamma_{0}, \sigma\right.$, and $\left.\xi\right)$ on eigenfrequencies were investigated for Model A, both CUS and CAS configurations.

(1) Preset Angle, $\alpha_{0}$. After calculating the eigenfrequencies via preset angles $\alpha_{0}$, the results are shown in Figure 12. Generally, from the results, the preset angle effects on the eigenfrequencies are very insignificant.

(2) Pretwist Angle, $\gamma_{0}$. Also, after calculating the eigenfrequencies via pretwist angles $\gamma_{0}$, the results are shown in Figure 13. As a result, lower-order eigenfrequencies were not almost affected by the pretwist angle but the higher modes increase the effects which is associated with the preset angles being higher.

(3) Taper Ratio, $\sigma$. Next, the eigenfrequencies via taper ratios $\sigma$ of the beam were found. The results are shown in Figure 14. In this case, the taper ratio has more effect on 
TABle 5: Stiffness matrix $\left[K_{p q}\right]$ of Model A and Model C.

(a)

\begin{tabular}{|c|c|c|c|c|c|c|c|}
\hline \multicolumn{8}{|c|}{ CUS: $\alpha_{0}=0$ and $\gamma_{0}=0$} \\
\hline$p$ & $q$ & 1 & 2 & 3 & 4 & 5 & 6 \\
\hline 1 & & $S$ & & & $S$ & $S$ & $A$ \\
\hline 2 & & & $S$ & & $S$ & $S$ & \\
\hline 3 & & & & $S$ & & $A$ & $S$ \\
\hline 4 & & & & & $S$ & $S$ & $A$ \\
\hline 5 & & & & & & $S$ & $A$ \\
\hline 6 & & & & Sym & & & $S$ \\
\hline
\end{tabular}

(b)

\begin{tabular}{lllccccc}
\hline \multicolumn{7}{c}{ CAS: $\alpha_{0}=0$ and $\gamma_{0}=0$} \\
\hline$p$ & $q$ & 1 & 2 & 3 & 4 & 5 & 6 \\
1 & & $S$ & & $S$ & & $S$ & \\
2 & & $S$ & & $S$ & & $S$ \\
3 & & & $S$ & & $S$ & \\
4 & & & & $S$ & & $S$ \\
5 & & & & & $S$ & \\
6 & & & Sym. & & $S$ \\
\hline
\end{tabular}

(c)

\begin{tabular}{|c|c|c|c|c|c|c|c|}
\hline \multicolumn{8}{|c|}{ CUS: $\alpha_{0} \neq 0$ or $\gamma_{0} \neq 0$} \\
\hline$p$ & $q$ & 1 & 2 & 3 & 4 & 5 & 6 \\
\hline 1 & & $S$ & $S$ & & $S$ & $S$ & $A$ \\
\hline 2 & & & $S$ & & $S$ & $S$ & $A$ \\
\hline 3 & & & & $S$ & $A$ & $A$ & $S$ \\
\hline 4 & & & & & $S$ & $S$ & $A$ \\
\hline 5 & & & & & & $S$ & $A$ \\
\hline 6 & & & & Sym. & & & $S$ \\
\hline
\end{tabular}

(d)

\begin{tabular}{llllllll}
\hline \multicolumn{7}{c}{ CAS: $\alpha_{0} \neq 0$ or $\gamma_{0} \neq 0$} \\
\hline$p$ & $q$ & 1 & 2 & 3 & 4 & 5 & 6 \\
1 & $S$ & $S$ & $S$ & $S$ & $S$ & $S$ \\
2 & & $S$ & $S$ & $S$ & $S$ & $S$ \\
3 & & & $S$ & $S$ & $S$ & \\
4 & & & & $S$ & $S$ & $S$ \\
5 & & & & & $S$ & $S$ \\
6 & & & & & & & $S$ \\
\hline
\end{tabular}

the eigenfrequencies related to the higher modes. The first and second eigenfrequencies decrease which is associated with the taper ratio being higher and the third or more eigenfrequencies increase along the taper ratio being higher.
Table 6: Mass matrix $\left[M_{p q}\right]$ of Model A and Model C.

(a)

\begin{tabular}{|c|c|c|c|c|c|c|c|}
\hline \multicolumn{8}{|c|}{ CUS \& CAS: $\alpha_{0}=0$ and $\gamma_{0}=0$} \\
\hline$p$ & $q$ & 1 & 2 & 3 & 4 & 5 & 6 \\
\hline 1 & & $S$ & & & & & \\
\hline 2 & & & $S$ & & & & 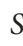 \\
\hline 3 & & & & $S$ & & $S$ & \\
\hline 4 & & & & & $S$ & & $S$ \\
\hline 5 & & & & & & $S$ & \\
\hline 6 & & & & Sym & & & $S$ \\
\hline
\end{tabular}

(b)

\begin{tabular}{|c|c|c|c|c|c|c|c|}
\hline \multicolumn{8}{|c|}{ CUS \& CAS: $\alpha_{0} \neq 0$ or $\gamma_{0} \neq 0$} \\
\hline$p$ & $q$ & 1 & 2 & 3 & 4 & 5 & 6 \\
\hline 1 & & $S$ & & & & & $S$ \\
\hline 2 & & & $S$ & & & & $S$ \\
\hline 3 & & & & $S$ & $S$ & $S$ & \\
\hline 4 & & & & & $S$ & $S$ & $S$ \\
\hline 5 & & & & & & $S$ & $S$ \\
\hline 6 & & & & Sym. & & & $S$ \\
\hline
\end{tabular}

(4) Section Ratio, $\xi$. Finally, the eigenfrequencies via section ratios $\xi$ of the beam were computed. The results are shown in Figure 15.

Figure 15 shows that the eigenfrequencies via section ratios have irregular trends for each. This is because of the characteristic that circles, squares, etc. have no values for primary warping functions [1], that is, see Figure 16, the primary function of the ellipse contour of Model A has almost zero values at $\xi=0.7716$. As a result, the effects of section ratio on the eigenfrequencies exist but the substantial factor of the effects is not section ratios but contour shapes.

3.4.5. Comparison of Model $B$ and Model C. For the comparison with a symmetric section, when $a=c$ in Model A, Model $\mathrm{A}$ is fixed to Model B (Figure 7). And then, the eigenfrequencies of Model B and Model C were compared to each other. The results are shown in Figure 17.

In Figure 17, Model $\mathrm{C}$ has symmetric eigenfrequencies at a ply angle of $90^{\circ}$, but on the contrary, Model $\mathrm{B}$ has not. Also, it is notable that CUS and CAS have the same values at ply angles $0^{\circ}, 90^{\circ}$, and $180^{\circ}$ for each, and especially, $\omega_{2}$ of CAS of Model B has an observable asymmetric curve of eigenfrequency which is one of vertical transverse shear vibration modes.

\section{Conclusion}

A lot of effort and attention are needed in deriving and correcting the warping functions from the geometric relationship 

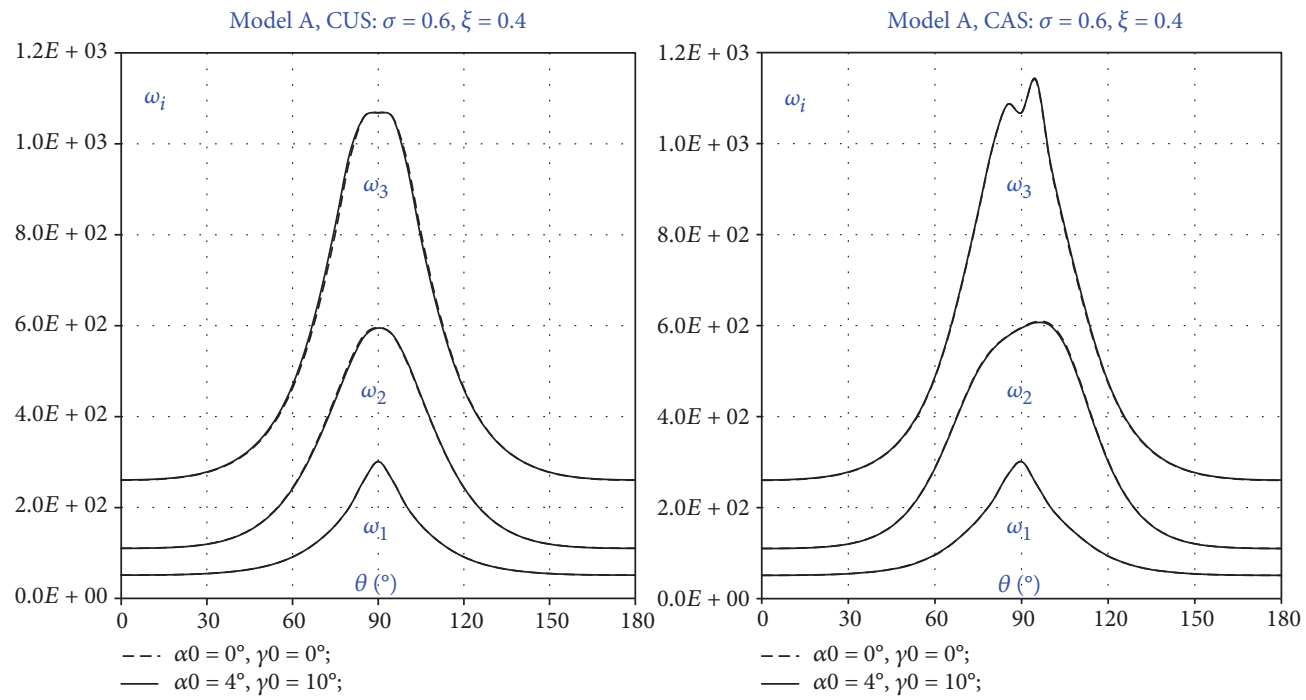

Figure 10: Eigenfrequencies vs. ply angle of Model A via $\alpha_{0}=0$ and $\gamma_{0}=0$ for CUS and CAS.

TABle 7: Eigenfrequencies of Model A via $\alpha_{0}=0$ and $\gamma_{0}=0$ for CUS and CAS.

\begin{tabular}{lcccccc}
\hline CUS $\left(^{\circ}\right)$ & \multicolumn{2}{c}{$\omega_{1}(\mathrm{rad} / \mathrm{s})$} & \multicolumn{2}{c}{$\omega_{2}(\mathrm{rad} / \mathrm{s})$} & \multicolumn{2}{c}{$\omega_{3}(\mathrm{rad} / \mathrm{s})$} \\
$\theta$ & $\alpha_{0}=0, \gamma_{0}=0$ & $\alpha_{0}=4, \gamma_{0}=10$ & $\alpha_{0}=0, \gamma_{0}=0$ & $\alpha_{0}=4, \gamma_{0}=10$ & $\alpha_{0}=0, \gamma_{0}=0$ & $\alpha_{0}=4, \gamma_{0}=10$ \\
\hline 0 & 51.1874 & 51.2014 & 110.305 & 110.096 & 260.03 & 260.587 \\
45 & 63.8732 & 63.9046 & 150.383 & 149.814 & 324.784 & 326.814 \\
90 & 301.001 & 301.106 & 595.863 & 594.734 & 1068.34 & 1068.17 \\
135 & 63.8728 & 63.8881 & 150.383 & 150.215 & 324.785 & 324.6 \\
180 & 51.1874 & 51.2014 & 110.305 & 110.096 & 260.03 & 260.587 \\
\hline 0 & 51.1874 & 51.2008 & 110.305 & 110.096 & 260.03 & 260.57 \\
45 & 64.6077 & 64.8452 & 159.239 & 158.803 & 328.334 & 329.249 \\
90 & 301.001 & 301.106 & 595.863 & 594.734 & 1068.34 & 1068.17 \\
135 & 63.9356 & 63.6511 & 161.385 & 160.896 & 324.406 & 325.731 \\
180 & 51.1874 & 51.2008 & 110.305 & 110.096 & 260.03 & 260.57 \\
\hline
\end{tabular}
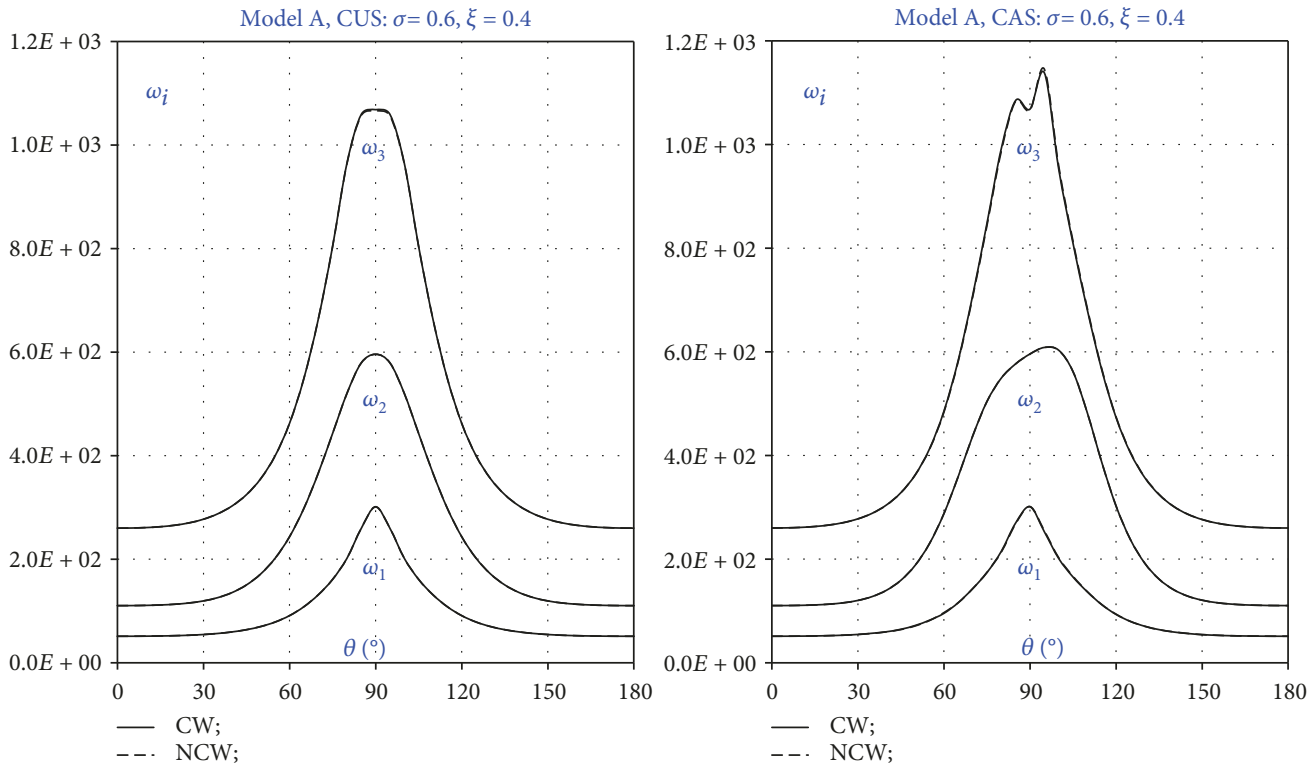

Figure 11: Eigenfrequencies vs. ply angle of Model A via CW and NCW for CUS and CAS. 
TABLE 8: Eigenfrequencies of Model A via CW and NCW for CUS and CAS.

\begin{tabular}{|c|c|c|c|c|c|c|}
\hline \multirow{2}{*}{$\begin{array}{l}\operatorname{CUS}\left({ }^{\circ}\right) \\
\theta\end{array}$} & \multicolumn{2}{|c|}{$\omega_{1}(\mathrm{rad} / \mathrm{s})$} & \multicolumn{2}{|c|}{$\omega_{2}(\mathrm{rad} / \mathrm{s})$} & \multicolumn{2}{|c|}{$\omega_{3}(\mathrm{rad} / \mathrm{s})$} \\
\hline & CW & NCW & CW & NCW & CW & NCW \\
\hline 0 & 51.1874 & 51.1852 & 110.305 & 110.303 & 260.03 & 260.026 \\
\hline 45 & 63.8732 & 63.8757 & 150.383 & 150.38 & 324.784 & 324.81 \\
\hline 90 & 301.001 & 301.359 & 595.863 & 595.858 & 1068.34 & 1065.79 \\
\hline 135 & 63.8728 & 63.8744 & 150.383 & 150.381 & 324.785 & 324.812 \\
\hline 180 & 51.1874 & 51.1852 & 110.305 & 110.303 & 260.03 & 260.026 \\
\hline 0 & 51.1874 & 51.1852 & 110.305 & 110.303 & 260.03 & 260.026 \\
\hline 45 & 64.6077 & 64.0606 & 159.239 & 159.237 & 328.334 & 328.122 \\
\hline 90 & 301.001 & 301.362 & 595.863 & 595.858 & 1068.34 & 1065.83 \\
\hline 135 & 63.9356 & 63.7094 & 161.385 & 161.381 & 324.406 & 325.027 \\
\hline 180 & 51.1874 & 51.1844 & 110.305 & 110.303 & 260.03 & 260.028 \\
\hline
\end{tabular}
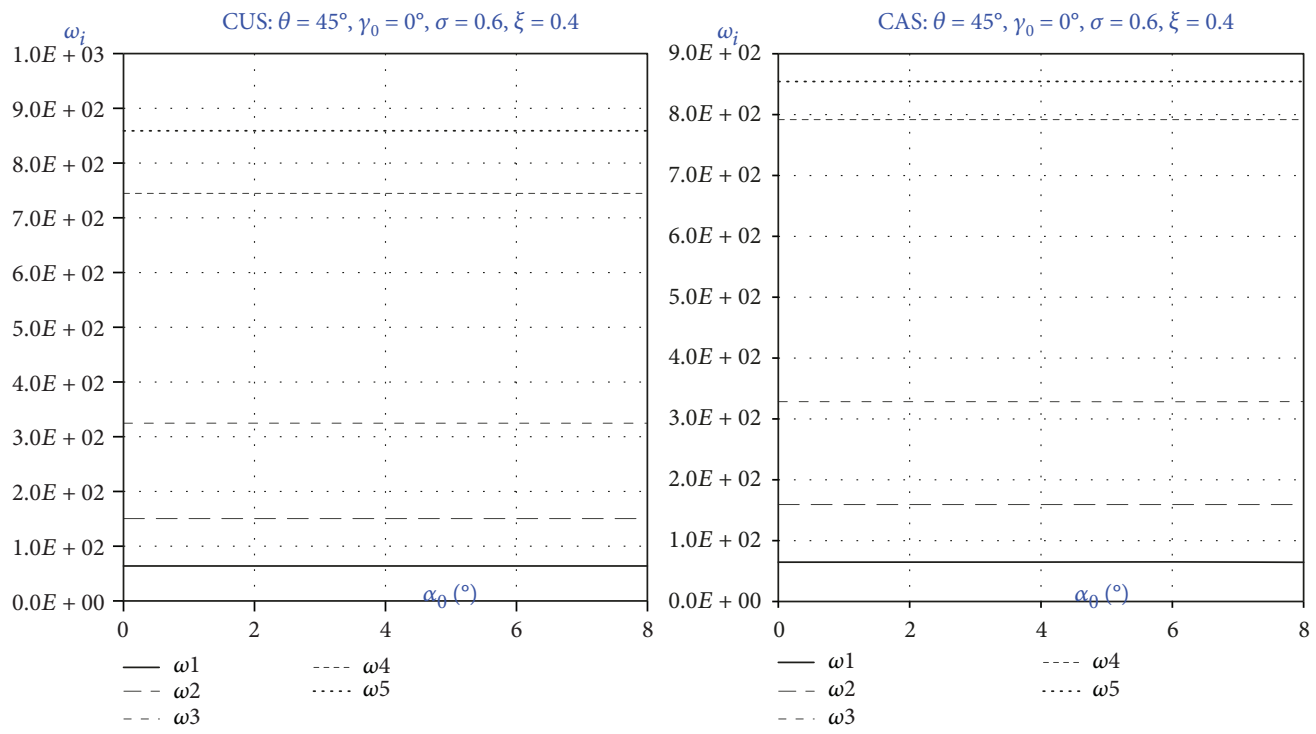

Figure 12: Eigenfrequencies vs. ply angle of Model A via $\alpha_{0}=0$ for CUS and CAS.
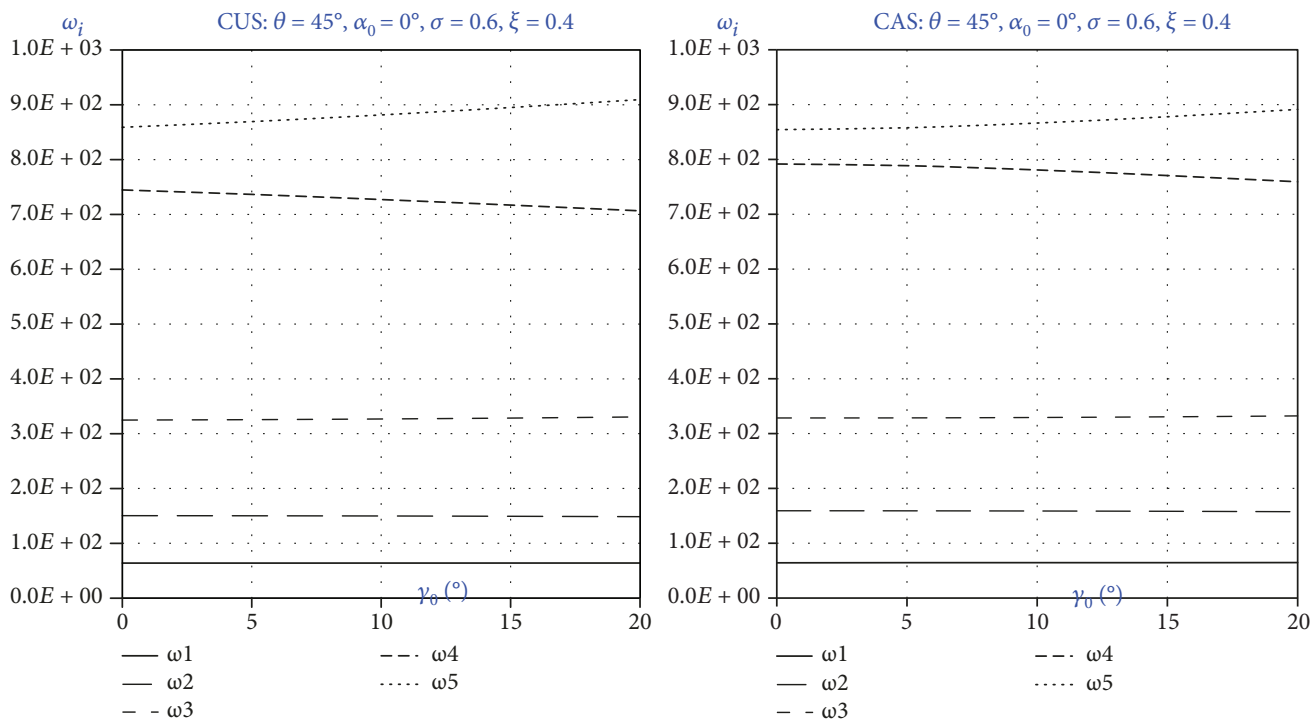

FIgURE 13: Eigenfrequencies vs. ply angle of Model A via $\gamma_{0}=0$ for CUS and CAS. 

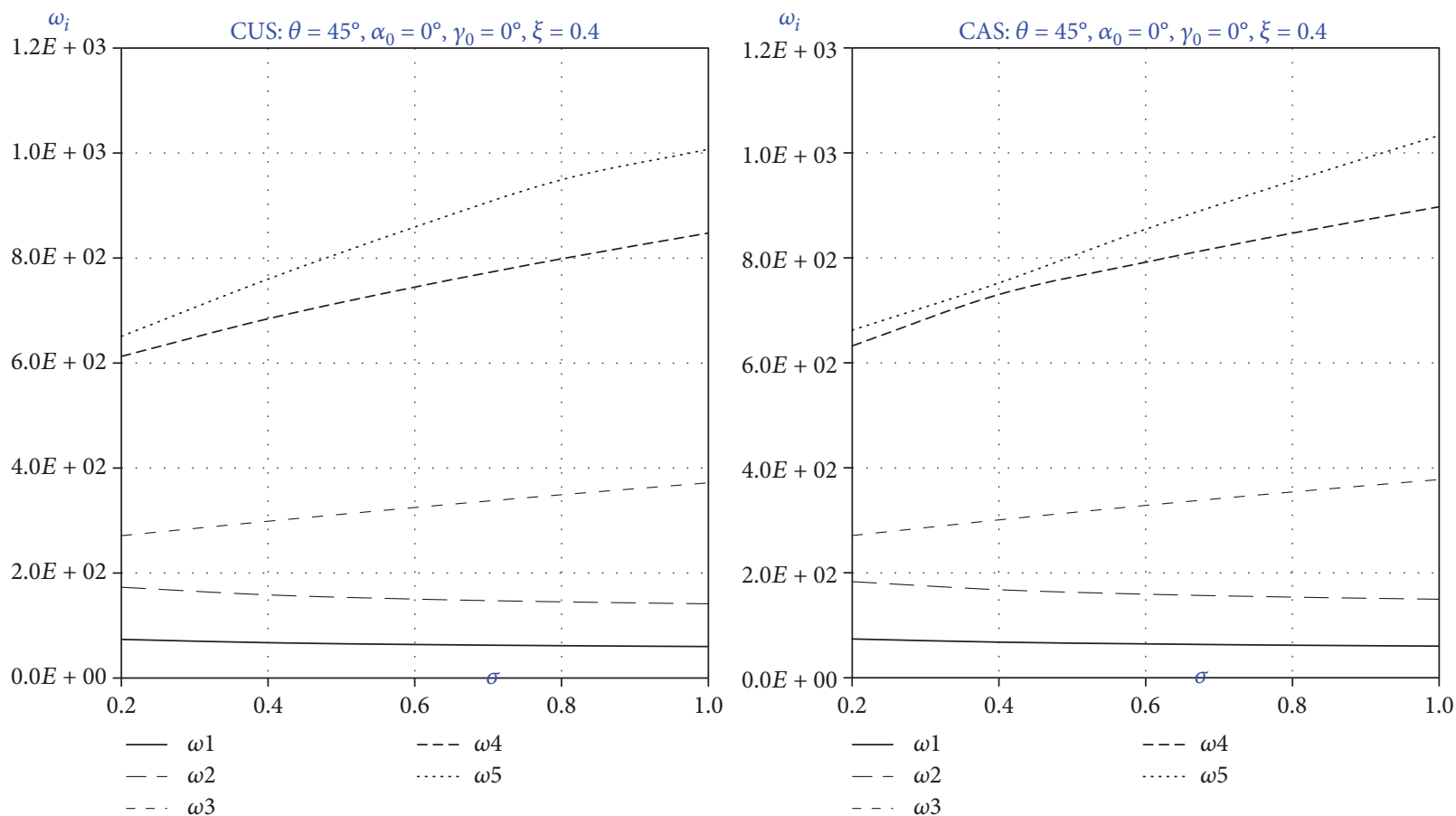

FIGURE 14: Eigenfrequencies vs. ply angle of Model A via $\sigma$ for CUS and CAS.
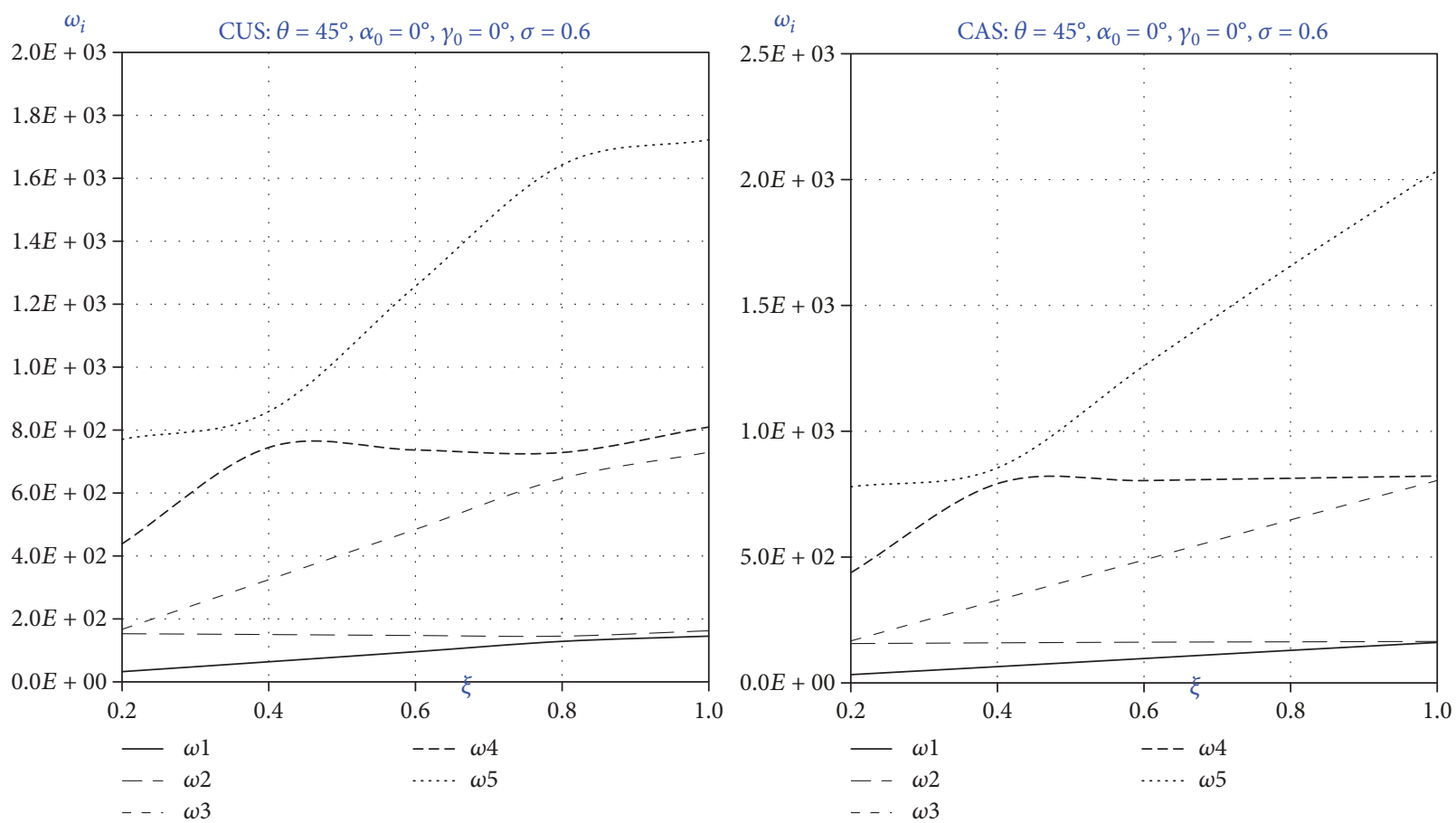

FIgURE 15: Eigenfrequencies vs. ply angle of Model A via $\xi$ for CUS and CAS.

of the chordwise asymmetric closed cross-sections. However, this is an indispensable procedure for initial sizing through analytical methods in the early stages of design development, such as aircraft wings and rotor blades.

For this purpose, in this paper, a model with a chordwise asymmetric closed cross-section (Model A) of a composite thin-walled beam was selected considering the contour shape for generalization of chordwise asymmetric closed cross-sections. And then, mathematical modeling was carried out by modifying the warping functions of the asymmetric cross-section and considering the transverse shear effect, warping restraint effect, and geometrical parameters, such as preset angle $\left(\alpha_{0}\right)$, pretwist angle $\left(\gamma_{0}\right)$, taper ratio $(\sigma)$, and section ratio $(\xi)$ of the beam. 


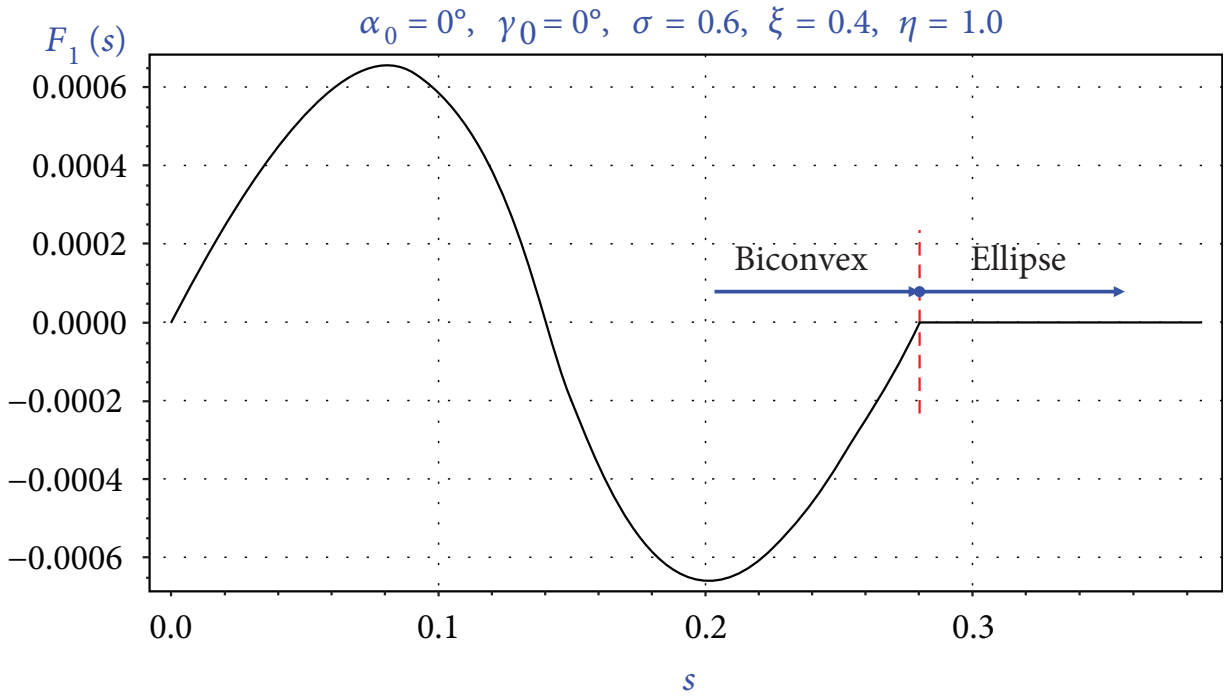

Figure 16: Primary warping function of Model A at $\xi=0.7716$.
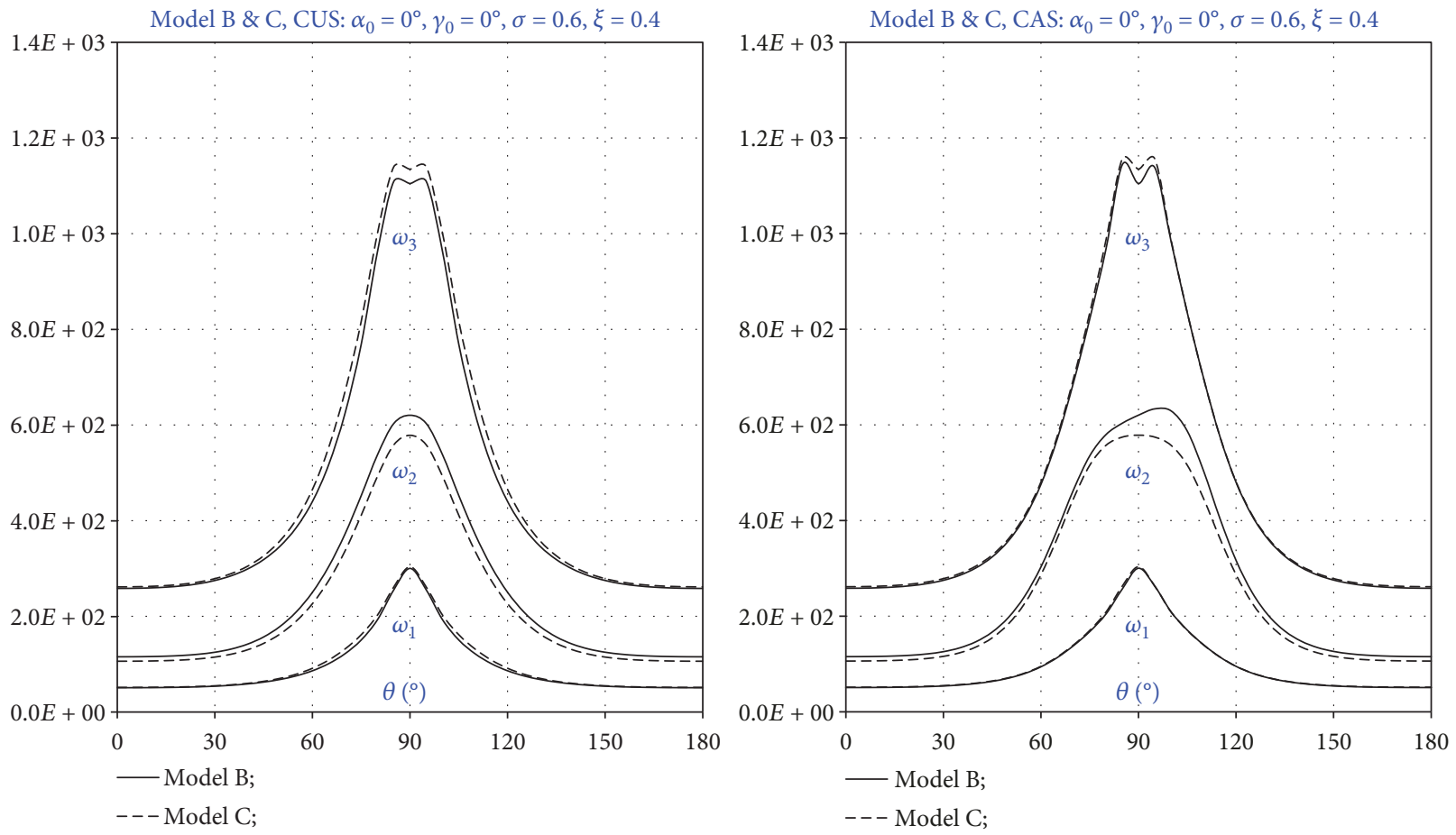

Figure 17: Eigenfrequencies of Model B and Model C for CUS and CAS.

Based on the mathematical modeling with the corrected warping function, mass coefficients, stiffness coefficients, and eigenfrequencies of the beam with a chordwise asymmetric closed cross-section were investigated for the CUS and CAS configurations of Model A. In addition, the present paper examined the effects of the following parameters: preset angle, pretwist angle, taper ratio, and section ratio of the beam. The results are presented as families of curves and tables, which may be used for the direct estimation of the eigenfrequencies for any given beam. Also, a comparative study was conducted on the symmetrical cross-section model (Model C) and the symmetrical cross-section model
(Model B) with same chord length and section thickness and the results were also provided.

From the above results, it is shown that the theoretical explicit analysis of the thin-walled beam with chordwise asymmetric closed cross-sections through the correction technique of the warping functions proposed in this study is possible and can be applied to the field of dynamic characteristics and aeroelasticity and so on. As a future research work, other lay-up schemes which may yield different elastic couplings might be considered. Moreover, in order to obtain more practical results, the multicell theory as well as stiffeners (spars and ribs) of the wing should be considered. 


\section{Data Availability}

The data used to support the findings of this study are available from the corresponding author upon request.

\section{Conflicts of Interest}

The author declares that there are no conflicts of interest regarding the publication of this paper.

\section{Acknowledgments}

This study has been performed for the Disaster and Public Safety Multicopter Development Program (SN: 10080047) funded by the Republic of Korean Government.

\section{References}

[1] L. Librescu and O. Song, Thin-Walled Composite Beams: Theory and Application, vol. 131 of Solid Mechanics and its Applications, , Springer, 2006.

[2] O. Song, Modeling and Response Analysis of Thin-Walled Beam Structures Constructed of Advanced Composite Materials, [Ph.D. Thesis], Virginia Polytechnic Institute and State University, 1990.

[3] L. Librescu, L. Meirovitch, and O. Song, "Integrated structural tailoring and control using adaptive materials for advanced aircraft wings," Journal of Aircraft, vol. 33, no. 1, pp. 203-213, 1996.

[4] S. Na, Control of Dynamic Response of Thin-Walled Composite Beams Using Structural Tailoring and Piezoelectric Actuation, [Ph.D. Thesis], Virginia Polytechnic Institute and State University, 1997.

[5] O. Song and L. Librescu, "Free vibration of anisotropic composite thin-walled beams of closed cross-section contour," Journal of Sound and Vibration, vol. 167, no. 1, pp. 129-147, 1993.

[6] Z. Qin and L. Librescu, "Static and dynamic validations of a refined thin-walled composite beam model," AIAA Journal, vol. 39, no. 12, pp. 2422-2424, 2001.

[7] S. Na and L. Librescu, "Optimal dynamic response control of elastically tailored nonuniform thin-walled adaptive beams," Journal of Aircraft, vol. 39, no. 3, pp. 469-479, 2002.

[8] S. Na, H. Yoon, and L. Librescu, "Effect of taper ratio on vibration and stability of a composite thin-walled spinning shaft," Thin-Walled Structures, vol. 44, no. 3, pp. 362-371, 2006.

[9] K.-T. Kim and O. Song, "Aileron reversal of nonuniform and swept composite aircraft wings," Journal of Aircraft, vol. 50, no. 3, pp. 681-693, 2013. 


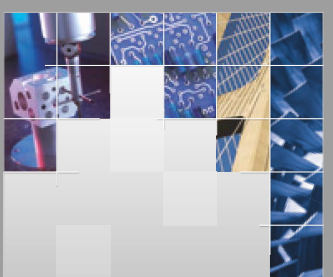

\section{Enfincering}
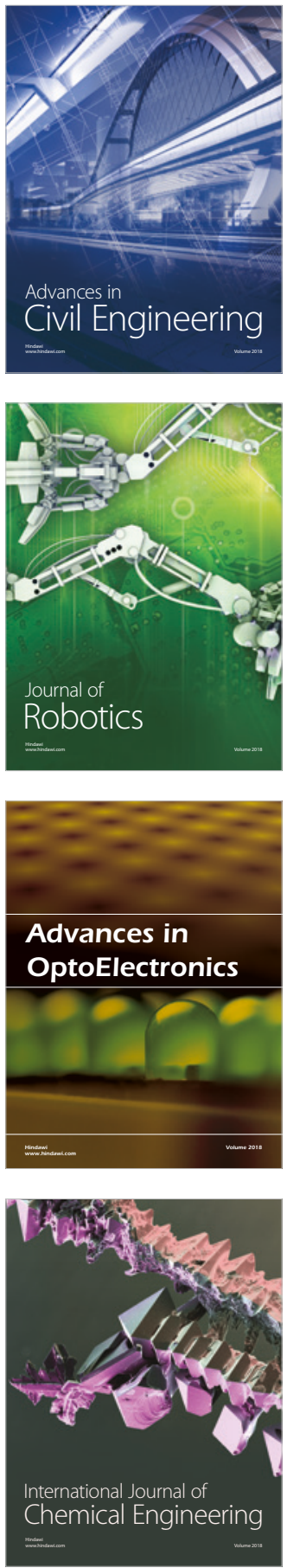

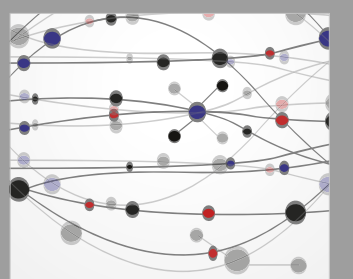

\section{Rotating \\ Machinery}

The Scientific World Journal

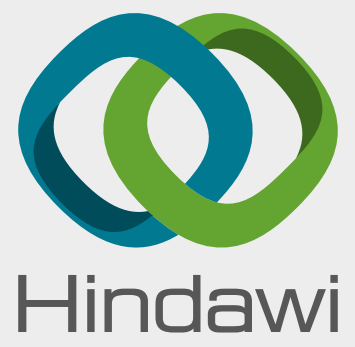

Submit your manuscripts at

www.hindawi.com
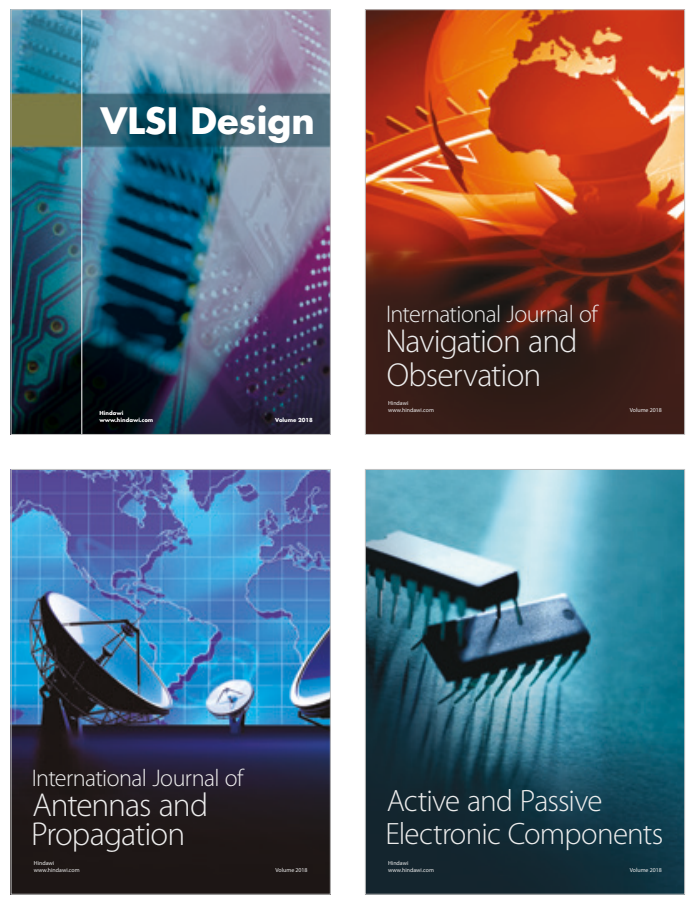
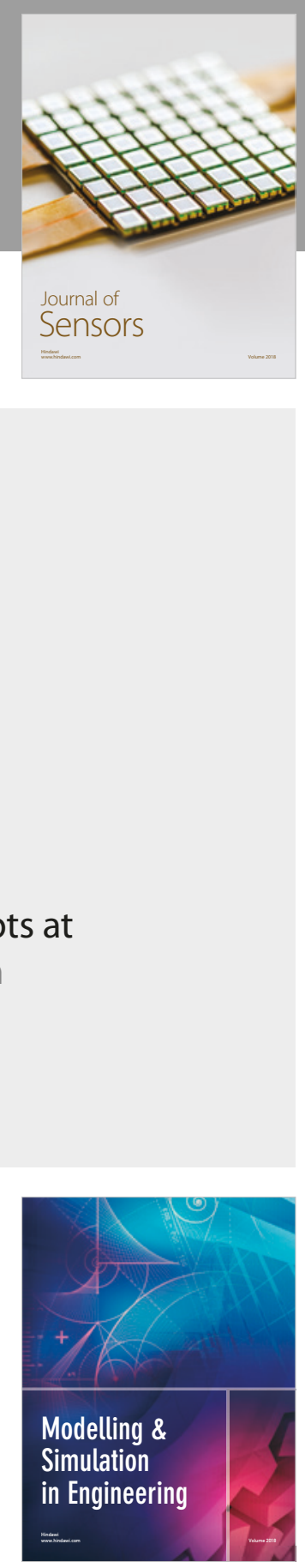

\section{Advances \\ Multimedia}
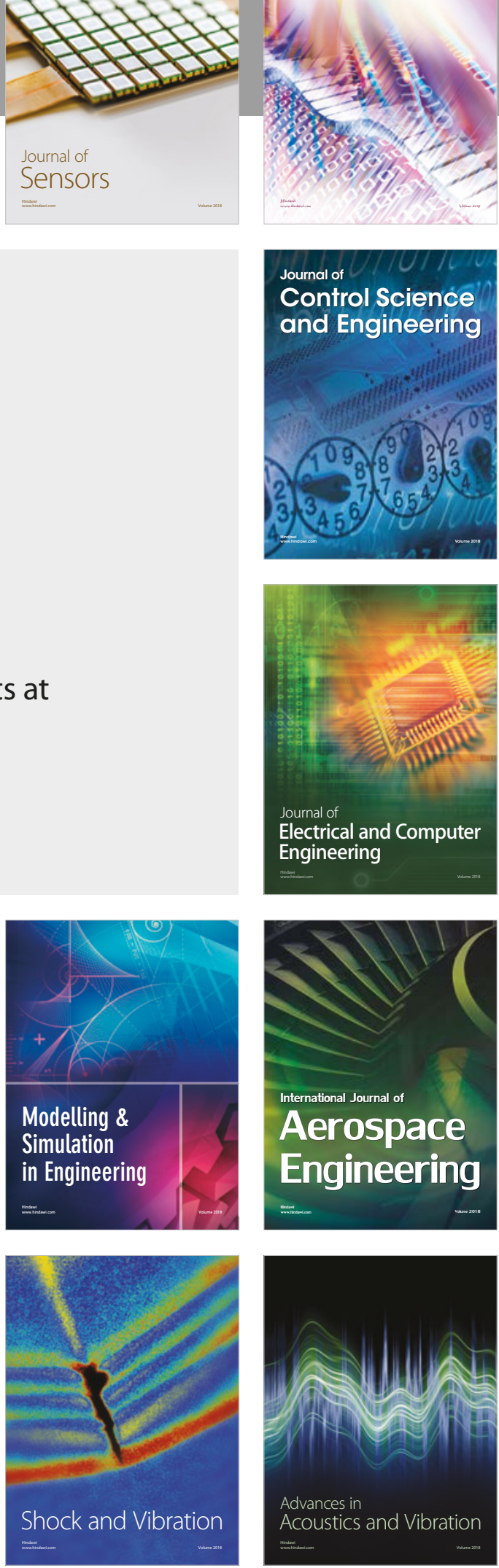